\title{
Chilodonella uncinata - As Potential Protozoan Biopesticide \\ for Mosquito Vectors of Human Diseases
}

\author{
Bina Pani Das ${ }^{1,2 *}$ \\ ${ }^{1}$ Former Joint Director, National Centre for Disease Control (NCDC), India, \\ ${ }^{2}$ Former Principal Investigator, DST Project, Jamia Millia Islamia (JMI), University, India \\ *Corresponding Author: Bina Pani Das, Former Joint Director, National Centre for Disease Control (NCDC), India, Former Principal \\ Investigator, DST Project, Jamia Millia Islamia (JMI), University, India.
}

Received: October 28, 2019; Published: November 11, 2019

DOI: 10.31080/ASMI.2019.02.0435

\begin{abstract}
Use of microbial control agents provide alternative method to synthetic pesticide for adequate insect management. Naturally occurring microorganisms such as viruses, bacteria, fungi and protozoa are used as biopesticides. Earlier studies revealed among entomopathogenic protozoa two ciliates, viz.: Lambornella clarki and Chilodonella uncinata are known with many biological control properties.

This article addresses the current status of development of Ch uncinata sand formulation which is available in dormant stage in easy formulation and packed in sachet as "infusion bag" (easy to store, transport and treat with shelf life $>18$ months) to be used as potential biopesticide for mosquito vector of human diseases.

During the process there were many questions like: (1) Ch uncinata culture should not be kept in refrigerator. But formulation prepared using the same culture tolerant to extreme cool weather. Reason: Ch uncinata has 4 stages in life cycle of which Trophont, the free living infective stage (in culture) is sensitive to cold; Cyst, the dormant stage (in the formulation) is tolerant to extreme cold situation. (2) Without artificial nutrition medium the culture remains in good condition for a few days when kept in Sun. Ch uncinata (trophont stage) has a chlorophyll particle in its body. (3) All the colonized mosquito larvae were highly susceptible to freshly prepared Ch uncinata strain at VCRC. Later these larvae at VCRC were found highly resistant to the same strain (sub-cultured repeatedly for a month). Due to repeated sub-culturing, Ch uncinata had lost their virulence. This was perhaps the same reason when a graduate student failed to induce infection in colonized larvae when both Ch uncinata and Culex pipiens were reared together. The student came out with a publication stating that $C x$ pipens was resistant to Ch uncinata which was found to be pathogenic to mosquito larvae elsewhere.

Keywords: Microbial Control Agents; Bacterial, Viral, Protozoan Biopesticides; Entomopathogenic Protozoa; Chilodonella uncinata; Lambornella clarki; Biological Control
\end{abstract}

\section{Introduction}

Mosquitoes act as vectors for most of the life threatening diseases like malaria, Japanese encephalitis, yellow fever, dengue fever, Chikungunya fever, filariasis, West Nile Virus infection, etc. Mosquito-borne diseases are endemic in more than over 100 countries around the world and mosquitoes alone transmit diseases to more than 700 million people annually [1]. In order to achieve immediate results in the control of mosquitoes sustained and prolonged use of synthetic chemical insecticides such as organochlorine and organophosphate compounds against vector mosquitoes for about four decades has led to the development of insecticide resistance in the target mosquito population, severe suppression of non-target organisms and general pollution of the environment [2]. Therefore, alternative and innovative vector control strategies are required either alone or in combination with traditional approaches, to decrease the transmission of the disease [3]. Microbial control agents provide an alternative to synthetic pesticides because of their generally low environmental pollution, low toxicity to humans, and other advantages [4]. Microorganisms that are naturally occurring (viruses, bacteria, fungi, protozoa) or the toxins produced by these organisms are used as biopesticides.

Entomopathogenic protozoa are a group of protozoa causing death to insect. They are unicellular eukaryotic organisms having size ranging from $30-75 \mu \mathrm{m}$ in length. They live parasitically inside the infected insect host hence they are also termed as endoparasites protozoa. More than 300 known species of entomopathogenic protozoa have been isolated from mosquitoes and other medically 
important arthropods. Most of these entomopathogenic protozoa are represented by Microsporidia and Ciliophora [5]. Microsporidians are all intracellular parasites. A few species belonging to Microsporidia are known as slow acting biopesticides causing low to moderate insect mortality over a period of weeks. Microsporidians were studied intensively yet there is no easy and cheap way to mass produce them in vitro. Hence research on their practical utility has been limited [6].

Only four species from Ciliophora (ciliates) are known to infect mosquitoes: they are: Lambornella stegomyiae, L clarki, Tetrahymena pyriformis and Chilodonella uncinata. All these species can cause mortality in mosquitoes and thus have potential as biocontrol agents. Of these, $L$ stegomyiae was the least studied ciliate that infect mosquito larvae. It was first discovered by Lamborn (1921) in Aedes albopictus collected from an old earthenware pot in Kuala Lumpur. Lamborn's preserved specimens were studied by Keilin (1921) who identified the ciliate as a new species and named it Lambornella stegomyiae [7].

A second ciliate species, L clarki was the most studied endoparasites ciliate isolated from tree hole-breeding mosquito larvae, Aedes sirensis in California, U.S.A [8]. The third ciliate, Tetrahymena pyriformis was observed in the body cavity and anal gills of several species bamboo-breeding mosquito Armigeres (Ar (Leicesteria) dolichocephalus, $\operatorname{Ar}(\mathrm{L})$ dentatus and $\mathrm{Ar}$ (L) digitatus) collected from bamboo forest near Kuala Lumpur [9]. This was followed by another observation under laboratory condition with four concentration of a clone of $T$ pyriformis against 2 species of colonized mosquito larvae, viz. Culex tarsalis and Aedes aegypti. Ae. aegypti larvae were found to be refractory to T. pyriformis infection producing only3.0\% mortality [10].

During mosquito larval survey (1999), author of this article accidentally discovered the fourth endoparasites ciliate, Chilodonella uncinata in the head capsule, antennae, body cavity, saddle, anal gills and siphon of mosquito larvae (Culex triateniorhynchus, $C x$. pseudovishnui, Cx. (Lutzia) sp, Anopheles stephensi mysoriensis and An hyrcanus group) breeding in paddy fields, irrigation channels, marshy areas, wells, ponds, and pools, in extensively paddy growing area of Sonepat district, Haryana, India. Subsequent follow up studies resulted in preparation of a protozoan biopesticide/biological control agent for mosquito vectors of human diseases [11]. As this was done first time in science, national and international patent applications were filed during 2001 on "A Microbial Control Agent for Mosquito Vectors of Human Diseases" in the name of DBT (Department of Biotechnology, Ministry of Science and Technology) and NCDC (National Centre for Disease Control), indicating author (Bina Pani Das) as "Inventor" [12]. Out of these four en- tomopathogenic protozoa, special emphasis is put on Ch uncinata because of its qualities like easy to colonize on artificial medium, mode of action, shelf life of formulation which is easy to store, transport and treat.

Events that lead to the invention leading to filing of national and international patent application

Step I of invention

JE vector survey

During August 1999, an inexperienced hand joined Bioassay laboratory of Medical Entomology and Vector Management, NCDC which was engaged in Japanese encephalitis vector survey. He was given practical demonstration of collecting mosquito larvae form natural breeding habitats as well as the precautions to be followed for transporting them to the laboratory alive. Next day he brought mosquito larvae from paddy fields of Safiabad village (Haryana) but by the time they were brought to lab all larvae died. He was told to repeat, however, again $100 \%$ mortality, prompted the author to detect the causative organism. Initial investigation only revealed very high mortality in natural population of JE vector larvae $(C X$. tritaeniorhynchus and Cx. pseudovishnui). About one tenth of these dead larvae were transparent and on examination under the microscope were found to be severely infected with endoparasites ciliates, which were found moving inside the entire body: haemocoel, head capsule, siphon, saddle and anal gills (Figure 1).

\section{Causative agent recognised}

As there was no expertise available in this field at NCDC, it took quite some time for excluding the commonly encountered, non pathogenic protozoan sharing the same aquatic habitat to recognize the causative agent responsible for repeated cent percent mortality in mosquito larvae in nature.

Isolation, colonization of candidate protozoan microbe under laboratory condition

These organisms were isolated from wild caught diseased mosquito larvae collected from paddy fields of Safiabad village. They were colonized under laboratory condition on artificial media using simple technique in glass container and filtered through $10 \mu \mathrm{m}$ mesh cloth. The culture filtrate was the first (basic) strain developed and these microbes though still not identified could be produced on large scale under laboratory condition (Temp. $27 \pm 2^{\circ} \mathrm{C}$ ), Humidity (70 - 80\%) within 48 - 72 hrs.

However, the basic culture strain developed at NCDC had to be sub cultured on alternate days to avoid contamination. After superannuating from NCDC (Dec 2007) culture procedure was modified in 2010 through the input collected from Biochemistry Department, 
South Campus, Delhi University thereby making it more efficient and standardised using the facilities available at the Department of Biosciences, Jamia Millia Islamia (JMI), a Central University, Delhi. The updated strain of Ch uncinata developed at JMI was deposited with the International Depository Authority (IDA) - ATCC, U.S.A. for which accession number: ATCC PRA-373 was allotted [13].

Ch. uncinata disperse in the environment by transovarian transmission

After the accidental detection of these causative agents responsible such severe mortality in 1999, wild caught female Cx. tritaeniorhynchus mosquitoes collected from the affected area were kept for egg laying separately under laboratory condition. Result indicated, the promising capability of this ciliate to get dispersed to newer areas as well as transovarian transmission capability (maternal transmission) of these ciliates from infected mosquito host to her offspring.

\section{Ch. uncinata can induce infection in colonized mosquito larvae}

Next series of experiments with wild caught infective larva as well as laboratory reared basic culture strain was able to induce infection in colonized mosquito larvae, viz. Anopheles stephensi (urban malaria vector), Aedes aegypti (vector of Dengue and Chikungunia) and Culex quinquefasciatus (vector of Filariasis).

\section{Ch. uncinata tolerant to desiccation}

Around this time, due to some unavoidable circumstances, author/inventor had to take 7 days off, keeping the small plastic containers with pure culture of basic strain of this ciliate unattended in her lab and on return these containers had to be re-flooded with distilled water as they were all dried up. To an utter surprise ciliates re-appeared in all the containers in 1 - 2 days time. That incidence prompted the inventor to run experiments under laboratory condition to substantiate the promising desiccation resistant property of the causative microbe (ciliate).

Causative microbe identified through help rendered by university of Delhi

During Oct 1999 guidance from the Protozoology Professor, Department of Zoology, University of Delhi was sought regarding identification of the said causative organisms in basic culture strain. He also helped by providing good photographs of $C x$. tritaeniorhynchus and $C x$ pseudovishnui larvae heavily infected with these endoparasites (Figure 1). One of his students carried with her sample of the basic culture strain while going to a Research Institute in Germany where these causative agents were identified by the experts in the field as Chilodonella uncinata (Ehrenberg), 1838 (Figure 2) (Subphylum: Ciliophora: Cryptophorida: Chilodonellidae) in wet mounts [14].
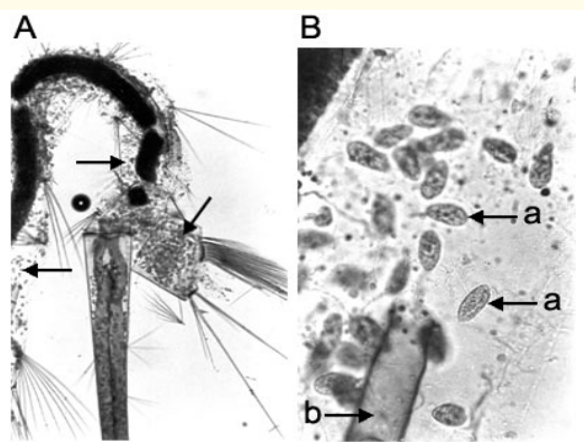

Figure 1: A, Cx tritaeniorhynchus larva: Arrow showing, endoparasites (microbe). B, a, endoparasites.

b, disintegrated alimentary canal (Source: Das 2003).

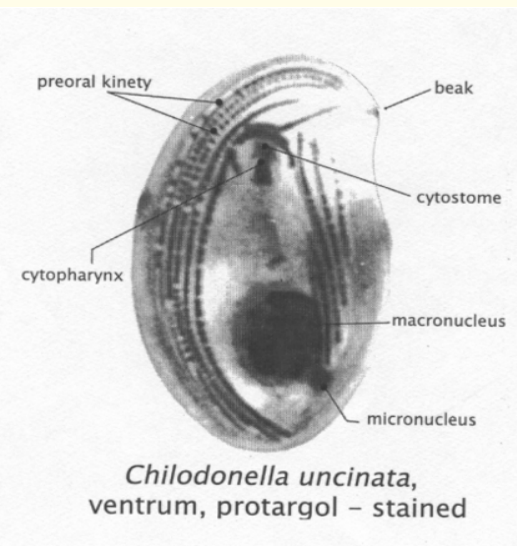

Figure 2: Free swimmingTrophont stage. (Source:US Patent \# 7141245, 2006).

\section{Guidance of Director of NCDC}

It was at this stage of development, the then Director, NCDC was apprised of the discovery. She was very happy but at the same time advised the inventor not to publicise and present the said new knowledge in any forum (workshop, conference, etc) and develop it further for the benefit of mankind. She also helped the author in literature survey as computer access at that time was available only to few senior level scientists. As per available literature, this was for the first time the genus Chilodonella was found to be pathogenic to mosquito larvae in nature. Out of 25 odd species worldwide, 3 species of Chilodonella, viz. Ch. cucullulus, Ch. rhesus and Ch. spiralidontis were reported earlier from India. Therefore, the discovery of the said entomopathogenic protozoan Ch. uncinata in Haryana was a new record for the species from India. 


\section{Preparation of Ch. uncinata formulation}

It took considerable time and follow up experiments to identify a suitable inert carrier, in the form of sterilized sand. The inert carrier was impregnated with known concentration of $\mathrm{Ch}$. uncinata culture strain, a semi-dry formulation was prepared in which the active ingredient (Ch uncinata) remain as encysted (dormant) form. The formulation when released in water works as an effective biological control agent for mosquito vectors of human diseases (Malaria, Japanese encephalitis, and Dengue/Chikungunya).

An. stephensi larvae were found to be more susceptible to protozoan formulation made out of basic culture strain. Initially the active ingredient (Ch uncinata) of the protozoan formulation was isolated from wild caught JE vector larvae. Thereafter, these were purified, colonized and when formulation was prepared it was found to be more effective against malaria vectors. The study clearly demonstrates how an event noted in the natural habitat can be brought to the laboratory refined and found to be a potential biopesticide capable of killing mosquito vectors of human diseases [15]. Formulation prepared using updated Ch uncinata strain developed at JMI was packed as "infusion bag" (Figure. 3) based on the wrapping paper used, was easy to store, transport and evaluate with a shelf life of $>18$ months [5]. It was made in different doses, viz.: $0.5 \mathrm{~g}, 1.0 \mathrm{~g}, 2.0 \mathrm{~g}, 4.0 \mathrm{~g}$ and $10.0 \mathrm{~g}$ [16].

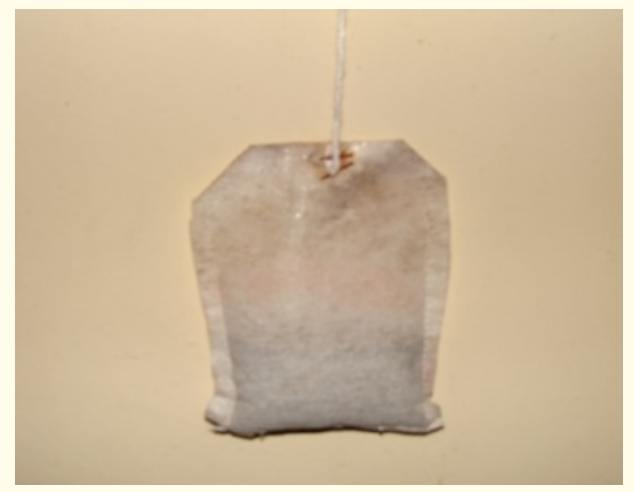

Figure 3: Infusion bag formulation of Chilodonella uncinata (Das 2017).

\section{Step II of invention}

Filing of patent application

Meeting with patent attorney

On request DBT agreed to support filing patent application covering the said invention and its subsequent maintenance. DBT appointed Patent Agent and Patent Attorney. Several discussions were held with Patent Attorney who was very much convinced with the merit of the said invention and advised that after filing the Indian patent application, should initiate the procedure for filing International patents through Patent Cooperation Treaty (PCT). The reason, he quoted for PCT filing, as granting of Indian patent application takes a very long time which unnecessarily creates wrong impression on the merit of the invention. On the contrary, International patent filing process takes much shorter time depending on excellent "search report".

\section{National/international patent applications filed}

Indian patent application entitled "a microbial control agent for mosquito vectors of human diseases" was filed on 08/08/2001. International patent applications were filed in six countries (U.S.A., Australia, Vietnam, Philippines, Bangladesh and Sri Lanka) through PCT route on 26.12.2001.

\section{International search report}

International search conducted by Austrian Patent Office, Vienna for the international patent application processed through PCT route related to this invention was very encouraging as the citations were of "A" category and patent application was allowed to enter National Phase directly under Chapter I based on "search report" without passing through examination i.e. under Chapter II.

\section{International patents granted}

On $1^{\text {st }}$ Aug 2008 (in about six and half yrs of filing international patent applications), six foreign countries granted patents related to the said invention. Whereas, for Indian patent application to be granted it took long 16 and half years. These countries with patent application \# and granting date are: Bangladesh (Patent \# 1003897, dt.08.08.2005); U.S.A. (Patent \# 7141245 dt. 28.11.2006); Australia (Patent \# 2002217423, dt.28.06.2007); Sri Lanka (Patent \# 13134, dt.11.12.2007); Vietnam (Patent \# 6774 dt. 31.12.07); Philippines (Patent \# 1-2003 - 500738 dt.01.08.08); India (Patent \# 292015, dt. 23.01.2018).

\section{Step III of invention}

Further study including Lab bioassay, field test

Mode of entry Ch. uncinata inside the host body and subsequent histopathology

Both expertise and facilities in this field of research were available at ICMR institute VCRC (Vector Control Research Centre), Puducherry. Under the guidance of experienced scientists at VCRC study on mode of entry of Ch. uncinata inside its host body and subsequent histopathology was carried out in 2005. VCRC colonized mosquito larvae (An stephensi). were treated with basic culture strain developed at NCDC and examined under microscope [13]. 
After $5 \mathrm{hrs}$ of post treatment a larva was slightly teased, instantly numerous endoparasites stage of $C h$. uncinata were found oozing out from the teased larva (Figure. 4A). After $48 \mathrm{hrs}$ all the larvae were dead, almost transparent and endoparasites stage of Ch. uncinata were found moving inside the entire host body (Figure. 4B). Entry points (shown by arrows) of the parasite on the cuticle of host body were also visible (Figure. 4C). Finally after few days Trophon stage of the parasite increased outside the larvae.
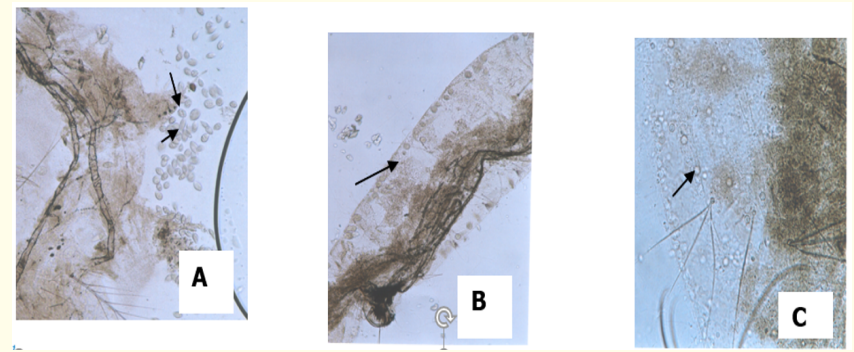

Figure 4: Mode of entry of Ch. uncinata inside host (An. stephensi) body. A, Teased larva after $5 \mathrm{hrs}$ of post treatment (arrows showing endoparasites). B, Another larva after a few days of post treatment. C, Entry points of parasites on host body [Source: Das 2012].

Microbial control agents as biopestisides

Utilization of Entomopathogenic Bacteria, Viruses, Protozoan, Fungi and Nematodes will require i) increased pathogen virulence and speed of kill, ii) improved pathogen performance under challenging environmental condition (cool weather, dry conditions, etc., iii) greater efficiency in their production, iv) improvement in the formulation that enable ease of application, increased environmental persistence, and longer shelf life, v) better understanding how they will fit into the system and their interaction with the environment and other integrated pest management (IPM) components, vi) greater appreciation of environmental advantages and vii) acceptance by farmers and general public [17].

One such microbial control agent with many biological control properties is Ch uncinata, a naturally occurring facultative protozoan parasite of $C x$ tritaeniorhynchus. Ch uncinata is desiccation resistant but sensitive to cool weather. However, it has adapted to tide over extreme cold climatic condition by way of transovarian transmission through its mosquito host in its area of influence in Northern India. During November, wild caught $C x$ tritaeniorhynchus females were predominantly unfed. From late Nov onwards as night temperature dropped in the area, female mosquitoes stopped taking blood meal and overwinters resting in available local vege- tation. Male population of $C x$ tritaeniorhynchus totally disappeared for next 3 cold winter months (December-February). From mid February due to gradual rise in night temperature, overwintering females started taking blood feed. If the mosquito happened to be infected and ovaries fully loaded with invaded endoparasites ciliate Ch uncinata which get dropped in water at the time mosquito mimics its egg laying habit.

The most successful microbial pesticide worldwide to date is Bacillus thuringiensis, which has dominated the microbial pesticide market. However, Bacillus thuringiensis var. israelensis (B.t.i) used in urban malaria control programme, does not recycle in the environment and its effect against surface feeding Anopheline larvae is impacted by various bioenvironmental factors, thus requiring weekly application in most habitats, increasing the end cost in the process [2]. Most viral biopesticides developed so far encounter difficulties as they are not stable under ultra violet rays of sun and more expensive than chemical pesticides due to in vivo production [6].

Unlike other biopesticides such as bacteria and viruses, Ch uncinata do not have to be ingested to cause infection to host mosquito larva, making them valuable as biological control agents. They enter the energy rich haemocoel (body cavity) of the host larva by drilling through the host cuticle. This type of mode of entry of Ch. uncinata from outside through host cuticle is similar to that reported in case of two parasitic ciliates, viz. L. stegomyiae and L. clarki and one parasitic fungi, Coelomomyces sp. However, Coelomomyces has a complex life cycle and requires both the primary host (mosquito larvae) and intermediate host (copepod) for reproduction [11]. $L$ clarki received considerable attention over the last two decades of the last century as a potential biological control agent for container breeding mosquitoes (Table 1 ). Parasite ( $L$ clarki)-induced dispersal by hosts, desiccation-resistant cysts, an active host-seeking infective stage, and high infection and mortality rates all indicate significant biological control potential for these and related ciliates against container-breeding mosquitoes [18]. Both $L$ clarki and $C h$ uncinata has many properties of a good biopesticide against mosquito vectors of human diseases (Table 1 ).

Laboratory evaluation with both culture strain and formulation of Ch uncinata was carried out at four institutes (VCRC, NCDC, JMI and ROSS) revealed efficacy of this protozoan (Ch uncinata) biopesticide is not dose dependent as least dose produced maximum mortality with minimum post exposure. Delayed development was noted in all colonized mosquito larvae exposed to Ch uncinata formulation. An stephensi larvae were most sensitive followed by $C x$ quinquefasciatus and Ae aegypti [5]. 


\begin{tabular}{|c|c|c|c|}
\hline \multirow{2}{*}{ Sl. No. } & \multirow{2}{*}{ Biological control property } & \multicolumn{2}{|c|}{ Entomopathogenic protozoa } \\
\hline & & L clarki & Ch uncinata \\
\hline 1 & Easy to colonize under laboratory condition & + & + \\
\hline 2 & Can be produced in large scale using simple technology & - & + \\
\hline 3 & Tolerant to desiccation & + & + \\
\hline 4 & $\begin{array}{l}\text { Robust and not sensitive to ultra violet radiation of sun and vagaries of agricul- } \\
\text { tural pesticides }\end{array}$ & - & + \\
\hline 5 & Facility to recycle in the environment & + & + \\
\hline 6 & Can pass from infected adult mosquito to offspring, & + & + \\
\hline 7. & Induces feeding inhibition and castration to mosquito host & + & + \\
\hline 8 & Not harmful to larvivorous fish & - & + \\
\hline 9 & Increased pathogen virulence and speed of kill & + & + \\
\hline 10 & Formulation easy to store, transport and treat; with $>18$ month shelf life & - & + \\
\hline 11 & $\begin{array}{l}\text { Susceptibility of laboratory reared mosquito larvae, An stephensi, Cx quinque- } \\
\text { fasciatus and Ae aegypti }\end{array}$ & - & + \\
\hline 12 & Natural host range & Ae sirensis & Wide host range $(*, \dagger, \ddagger, \S, \|, \rrbracket)$ \\
\hline
\end{tabular}

Table 1: Biological control properties of Lambornella clarki and Chilodonella uncinata

Source: (Das 2003; 2008; 2012; 2017; Das et al. 2016) (Egerter, et al. 1986)

* Culex tritaeniorhynchus † Cx pseudovishnui $\ddagger$ Cx (Lutzia) sp.§ Cx (Culex) sp. || Anopheles stephensi mysoriensis $\uparrow$ An hyrcanus gp.

Since 2013 onwards no more laboratory facility was available and the author of this article had to undertake a study on the use of infusion bag formulation of Ch. uncinata to control Ae. aegypti breeding in large water-storage tub kept in a courtyard of Delhi. Ae. aegypti larvae used in the study were those hatched from eggs laid by wild Ae aegypti mosquitoes during a period of nearly eight weeks from mid September 2016 to first week of November 2016 in a disused black plastic tray. The study revealed $30 \mathrm{~g}$ infusion bag formulation of this biopesticide provided 3 months control of Ae. aegypti in 40 liter domestic water storage tub, one of the most common and preferred larval habitat of this species in NCT, Delhi during dengue transmission that normally extends from August to November [16]. Later with the assistance of technical staff of South Delhi Municipal Corporation, infusion bag formulation was evaluated for its residual efficacy to control Ae aegypti breeding under field condition. It was observed that one time application of $80.0 \mathrm{~g}$ infusion bag formulation of Ch uncinata impacted $100 \%$ control of Ae aegypti breeding for 8.0 and 9.5 weeks in cooler and cemented tank respectively in a posh locality in Delhi during June to October coinciding with major period of Dengue transmission season in the city [19]. Chilodonella uncinata in dormant stage is available in a sand formulation and packed in a sachet (Infusion bag) which on dipping in affected water will revive the organism (the microbial agent) will kill pest mosquito larvae thereby controlling vector density. Ch uncinata has shown long-term effectiveness against immature stages of Ae aegypti under field condition during pre-monsoon and monsoon season. It appears to be one of the alternatives to conventional chemical insecticides such as tempeh's where Aedes larvae had been shown to develop resistance. In addition to Municipal Corporation, Ch. uncinata infusion bag formulation can also be used by individuals and community to control Aedes aegypti breeding in manmade water storage containers kept in their domestic and peri-domestic area [19].

Patent application for the technology developed by the author was filed in 7 countries including USA, Australia and India in 2001. These countries have already granted the said patent. In the past more than 18 years, even after retirement, the author has continued further research on the topic under lab and field conditions, with whatever support she could find from DST, JMI, ROSS and SDMC. If the necessary procedural requirements for introducing the same to the National Vector Borne Disease Control Program had been taken in time, the benefit of this simple and inexpensive technique would have accrued to the general public long ago.

\section{Conclusion}

This technology is most appropriate technology for all those countries where mosquitoes menace is rampant. Ch. uncinata is natural as well as indigenous and in areas with very high prevalence it is known to have control over mosquito vector of Japanese encephalitis in nature. Earlier studies on laboratory bioassay indicated that a lower dose of this protozoan formulation can be used 
as a potential biopesticide to reduce vectors of malaria, dengue/ Chikungunya and filariasis in the context of integrated vector management. Author remains hopeful that more extensive field trials and bio-safety studies involving industrial partners who can produce potent formulation and maintain required supply of the same for its application in public interest.

\section{Bibliography}

1. Jiang Y and Mulla MS. "Laboratory and field evaluation of spinosad, a biorational natural product, against larvae of $\mathrm{Cu}$ lex mosquitoes". Journal of Mosquito Control Association 25 (2009): 456-466.

2. Mittal PK. "Biolarvicides in vector control: Challenges and prospects". Journal of Vector Borne Diseases 40 (2003): 20-32.

3. Rojas W., et al. "Reduction of malaria prevalence after the introduction of Romanomermis culicivorax (Mermithidae: Nematoda) in larval Anopheles habitats in Colombia". Bulletin WHO 65 (1987): 331-337.

4. Liu SQ., et al. "Proceeding of Chinese Entomological Society Conference". Science and Technique Press, China (2000):10981104.

5. Das BP., et al. "Laboratory bioassay of Chilodonella uncinata, an entomopathogenic protozoan, against mosquito larvae". Journal of Mosquito Research 6 (2016):1-10.

6. Canon U. “Chapter 13. Microorganisms in Biological pest control - A Review (Bacterial Toxin Application and Effect Environmental Factors)". In: Marina Silva-Opps, (ed.), Current progress in Biological Research, Europe, In Tech, (2013) p. 287-317.

7. Lamborn WA. "A protozoan pathogenic to mosquito larvae". Parasitology 13 (1921): 213-215.

8. Corliss JO and Coats DW. "A new cuticular cyst producing tetrahymenid ciliate, Lambornella clarki n. sp. and the current status of ciliatosis in culicine mosquitoes". Transaction American Microscope Society 95 (1976): 725-739.

9. Corliss JO. "Natural infection of tropical mosquitoes by a ciliated protozoa of the genus Tetrahymena". Transaction Royal Society Tropical Medicine and Hygiene 55 (1961): 149-152.

10. Grassmick RA and Rowley WA. "Larval mortality of Culex tarsalis and Aedes aegypti when reared with different concentrations of Tetrahymena pyriformis". Journal of Invertebrate Pathology 22 (1973): 86-93.
11. Das BP. "Chilodonella uncinata - a Protozoa pathogenic to mosquito larvae". Current Science 85 (2003): 483-489.

12. Das BP. "Process for preparation of a microbial control agent". U.S. Patent US (2006): 7141245.

13. Das BP. "Chapter 5. Ecology of Culex tritaeniorhynchus in and adjoining areas of Delhi, non-endemic area in Northern India, with special reference to Chilodonella uncinata as a Bio-control agent, In: Das BP. Mosquito Vectors of Japanese encephalitis virus from Northern India, Role of BPD Hop Cage Method, Springer Briefs in Animal Sciences, New York (2012): 61-83.

14. Foissner W. "Basic light and scanning electron microscopic methods for taxonomic studies of ciliated protozoa". Europian Journal of Protistology 27 (1991): 313-330.

15. Das BP. "New microbial insecticide - a discovery by accident". Invention Intelligence 43 (2008): 26-28.

16. Das BP. "Three months of Aedes aegypti control with a novel infusion bag formulation of Chilodonella uncinata in domestic water-storage container in Delhi". International Journal of Mosquito Research 4.2 (2017): 102-107.

17. Lacey LA., et al. "Insect pathogens as biological control: Do they have a future?" Biological Control 21 (2001): 230-248.

18. Egerter DE., et al. "Dispersal of parasitic ciliate Lambornella clarki: Implication for ciliates in the biological control of mosquitoes". Proceedings of the National Academy of Sciences USA 83 (1986): 7335-7339.

19. Das BP and Tuli NR. "Field evaluation of Chilodonella uncinata in desert coolers and cemented tanks in Delhi". International Journal of Mosquito Research 6 (2019): 39-45.

\section{Volume 2 Issue 12 December 2019} (C) All rights are reserved by Bina Pani Das. 